\title{
Ordinary and Everywhere
}

Book review by Annebella Pollen, New Formations 79, 2013, pp. 159-161

Martin Hand, Ubiquitous Photography, Cambridge, Polity Press, 2012, 219pp; $£ 14.99$ paperback

Martin Hand's timely overview of the discourses, technologies and practices of digital photography draws on a range of approaches from the sociology of consumption, visual and media culture alongside science and technology studies to offer a useful introduction to the complexities of contemporary photographic debates, particularly in relation to popular practice. Hand argues that 'the confines of photography studies, with their primary focus on the visual image, are necessarily limited in their scope for understanding the broad dynamics of digitisation.' He suggests instead that such a study 'requires analyses that pay serious attention to the theory and history of photography but equally are able to move well beyond those boundaries.' (p4) While this assertion seems to indicate an insularity in photography studies that some would say misrepresents the broad and catholic nature of the field, in practice Hand's primary interest in how photographs are used, rather than their 'discursive, semiotic and material character' (p59), means that he doesn't actually examine images at all. There is certainly a persuasive case to be made that the camera 'has always been a relational device', even 'a node' embedded in larger social, cultural, technological and ideological networks (p132), and indeed that 'photographies are best understood as practices,' (p97) yet what gets pictured is clearly an integral part of the process of meaning-making. Subject matter is not arbitrary. Ubiquitous photography - rather than the gargantuan abundance of photographs, in all their complex specificity - is the principal concern here.

Despite this deliberate - but perhaps convenient - omission, there is no doubt that Ubiquitous Photography will function very well as a stimulating teaching tool and as an up-to-date primer for those new to the area. The book largely serves to synthesise, debate and challenge existing knowledge about the proliferation and pervasiveness of popular photographic practice, which it does very effectively and comprehensively. To a lesser extent, it also contributes new knowledge on the subject, in the form of empirical case studies that aim to examine 'the different and complex relationships between digital images and the material environments within which they are actually enacted and distributed' (p95). Research conducted for this book included content analysis of the entire back catalogue of Popular Photography magazine (1937-present) to investigate 'the emergence of digital photography as a recognizable practice', (p20) a social biography of the digital camera itself, and ethnographic interviews with a range of digital photographic consumers, from archivists to camera club members and undergraduate students. This material, disappointingly, is 
not always foregrounded in the book - indeed some results are hardly visible - and what is used sometimes serves merely to add colourful quotations, by way of illustration, to points already established through theoretical discussion. When this empirical material is highlighted, however, Hand makes striking observations that can enhance understanding of the effects of multiplication and diversification on contemporary photographic practices in everyday life.

For example, Hand's archival research reveals a pertinent parallel between technological shifts from analogue to digital photography in the late twentieth century and wet-plate to dry-plate processes in the late nineteenth century. In an amusing extract from a 1900 edition of Photographic News that suggests a technological determinism all too familiar in the many inflated claims made for digital photography, it is asserted, 'in photography, as in everything else, the fittest survives, and the fittest negative process is gelatino-bromide on glass, paper and film'. (p100) Anxieties around change are also mapped through more recent histories of popular photographic and computing literature. Here digital technology has shifted from being conceptualised as an indication of a medium in crisis (in relation to the autonomy of the photographer, for example) to a site of creative promise. Hand shows, through this archival material, how digital cameras, as a 'predatory technology', needed to be carefully ascribed with aspects of familiarity alongside their technical novelty in order to achieve popularity. He also links longer historical concerns about automation in photography - as a longstanding threat to art, for example - with more recent anxieties about digital automation as deskilling. Through examination of the different ways that digital photography has been described by the press (for example, as potentially undermining the precarious boundary between the 'serious' photographer and the casual snapper) and through the practices of its users (which demonstrate that digital cameras can afford experimentation, greater control and rapid build-up of tacit knowledge), Hand is able to balance a range of sources to overturn received wisdom.

Throughout, Hand aims to trace digital photography's complexity through its relationship to earlier photographies. This is a refreshing approach that tempers the occasionally apocalyptic and ahistorical claims made of photography's imminent demise at the advent of digital formats. While there are some radical departures and even cultural ruptures evident in the present photographic landscape, Hand seeks to locate precisely which practices are historically seamless and which dismantle and reassemble traditions. In most cases, Hand argues that there has largely been an 'intensification' of pre-existing photographic practices as a result of its mass-proliferation and massdistribution, but he also identifies important, unprecedented consequences of photography's new expansion into ubiquity. One of these concerns the routine 'overabundance' of individuals' personal photograph collections and the seemingly inexhaustible range of methods available for their storage 
and classification. In Chapter 5, in particular, Hand examines the effects that these new methods may have on memory making, and highlights the simultaneous fear of loss (of our precious, vulnerable photographs) with the fear of endurance (of resilient photos, archived forever online, that we would rather forget). Through his interviews with undergraduate students, Hand draws out some of the novel ethical challenges and 'affective responsibilities' bound up with photo-sharing practices that indicate a need for new models of living publicly in what he chillingly describes as a 'post-privacy world' (p183). Hand's student interviewees offer interesting insights into the changing effects of ubiquity on photography's perceived authenticity and value. Ephemerality is increasingly prized over durability, as demonstrated in the production and display of what might be called 'bad photographs'; 'the banal or in-between moments not normally included in edited or ordered narratives' (p92). Through these kinds of images, Hand argues, authenticity is manufactured and performed though the picturing of mundanity.

It is these tantalising micro-empirical studies of photography practice on the ground that are the most satisfying and original aspects of Hand's text; they are also, unfortunately, among the briefest. It is a shame that this new research was not given a more leading role. As digital photography is evidently so ubiquitous, broad theoretical claims made about its character and meaning inevitably risk generalisation. The specific material affordances of digitisation, as Hand wisely points out, 'are enacted differently in relation to established conventions... among different constituencies' (p94). Where Hand is able to examine these specific, localised cultural practices, he demonstrates digital photography's particularities and complexities rather than narrowing understanding of the form. The mixed methods of Hand's approach allow him to argue, convincingly, for the mutual photographic entanglement of technology and practice. Image analysis remains frustratingly absent as the obvious missing element that would triangulate - and potentially further problematise - these fascinating debates. 